\title{
The use of oncolytic viruses in selected tumours
}

\author{
Paweł Macieląg', Sylwia Foltyn', Małgorzata Polz-Dacewicz ${ }^{1}$ \\ ${ }^{1}$ Departament of Virology, Medical University, Lublin, Poland \\ Macieląg P, Foltyn S, Polz-Dacewicz M. The use of oncolytic viruses in selected tumours. J Pre-Clin Clin Res. 2013; 7(2): 98-103.
}

\begin{abstract}
Introduction. Neoplastic diseases are the leading cause of death among adults. Despite significant advances in pharmacotherapy, advanced radiotherapy and high level of oncology surgery, the effects of treatment are still too low. Doctors are attempting to find new, effective methods to cure people suffering from cancer. The big hope for a breakthrough in the treatment of cancer is placed in oncolytic viruses therapy. An innovative approach to viruses, usually associated with a potential risk to health, presents a chance for a breakthrough in oncology. This article is an overview of studies that have used different oncolytic viruses.

State of knowledge. Currently, a lot of research is being carried out on the use of viruses in oncology. The mechanism of action of this method is to infect the tumour cells with a virus, replicating inside them and their lysis during the release of new virions. An additional advantage is the possibility for use in many types of cancer and regardless of its clinical stage. Furthermore, virotherapy may be combined with standard methods for achieving greater results.

Summary. The results of these studies demonstrate the high effectiveness of this therapy. A large force action is combined with low side-effects and the ability to connect it with other therapies.
\end{abstract}

\section{Key words}

oncolytic viruses, oncolytic virotherapy, head and neck neoplasms, antineoplastic agents

\section{INTRODUCTION}

Viral infection is mainly associated with a potential threat to health and even life. Small viruses invisible even under the microscope, unable to expand beyond the living host cells, have a very high tropism for specific cell (receptors on the cell membrane) and easy replication mechanisms enabling their rapid reproduction. Some viruses, during the release of newly-created virions, cause lysis of the host cell. This potentially negative property was noticed at the beginning of the twentieth century and caused questing doctors to use viruses to treat cancer diseases. However, despite initially obtaining detailed test results, the final results did not meet the expectations of researchers and the development of oncolytic virus therapy has been stopped [1]. Great progress in biotechnology, molecular genetics and immunology allows the modification of the genetic material of viruses to increase tropism for cancer cells, reduce systemic toxicity and weaken the impacts of the immune system person treated with the use of oncolytic viruses. Current work includes the review of selected literature describing studies using oncolytic viruses where there is evident a great chance for a new, effective treatment for various types of cancer, with particular emphasis on those located within the head and neck.

Challenges of modern therapy. The mechanisms used in the treatment with viruses are dependent on the type of virus used in the test (treatment), and tumour type. Viruses can directly cause cell lysis, induce an immune response to cancer, sensitize tumours to chemotherapy and radiotherapy, lead to cytotoxicity by proteins produced during replication, and also serve as vectors of genes that cause cell apoptosis [2]. The development of cancer therapies using viruses has to

Address for correspondence: Paweł Macieląg, Departament of Virology, Medical University, Lublin, Poland

e-mail: pawel.macielag@wp.pl

Received: 01 August 2013; accepted: 21 February 2014 overcome many obstacles that limit its rapid progress; one of the major problems is the method of administration of the viruses. Intratumoral virus distribution is the optimal way of the dosage; however, this method is limited to easily accessible tumours. Other methods of administration have been tested in animal models and found to be an effective: intravenous, intraarterial and intraperitoneal. Initial concerns relating to the administration of these methods were unfounded. The systemic administration of a virus which is well tolerated, may significantly limit their therapeutic role. The organism may develop antibodies that neutralize viruses, thus significantly reducing the effects of treatment [3]. Great progress which has been made in the ability to make changes in the genetic material of viruses, causing an increase in the specificity for desirable tissues. There are two ways to increase the specificity:

- by modifying the viral receptor in order to connect with one specific molecule on the surface of tumour cells;

- removal of theviral genes responsible for the initiation of replication.

To proliferate in the cell, the virus needs a specific promoter, and their over-expression is found in modified tumour cells. Both methods increase the specificity. Due to the different points of the handle operation it is possible to combine one with the other to increasing the specificity of the virus. Improved tropism for certain tissue reduces the amount of virus entering the body. Still, the problem is to determine the effective dose of virus that will have a therapeutic effect and not elicit strong adverse reactions [4].

The use of virus therapy does not exclude standard therapy of cancers. Much of the work focuses on the synergistic treatment in which viruses are part of multi-therapeutic activities. Lysis of tumour cells could allow operations on previously unresectable tumours, and cause apoptosis of a tumour incompletely removed during surgery. The use of both types of therapy allows the use of smaller doses and thereby a lessening of side-effects [5]. 
The risk of an infection caused by viruses is very small, even in the case of people with an impaired immune system during cancer disease. Viral particles are often unable to infect healthy cells, and the problem can be excessive immune system response in the person being treated. During the treatment of overexpression of cytokine genes (GM-CSF, Il-12, etc.) due to ongoing local inflammatory process.

Unfortunately, the number of clinical trials in humans is still very low, and the majority of the studies are based on cell lines or animal organisms. In the first case, the role of the immune system is eliminated, while in the case of animal therapy, is well tolerated. Side-effects related to the treatment with oncolytics viruses are associated with a mild local inflammatory process induced by replicating virions, but systemic reactions are not observed [6]. Viruses also can boost the immune system towards anti-tumour responses. This is made by changing the receptors on cell membranes which sensitize cell on the reactions of the immune system. Another way is to increase the production of anti-cancer cytokines [7].

Head and neck cancers. Head and neck cancers account for at least $5.3 \%$ of the total malignancies recorded in Poland, including $8.3 \%$ in men and $1.9 \%$ in women. The incidence of head and neck cancer is therefore nearly five times higher in men than in women. Over $80 \%$ of these tumours are squamous cell carcinomas derived from the squamous epithelium that line the respiratory and oral tracts. The remaining cases are adenocarcinomas, lymphatic and transient cancers, melanomas, sarcomas and tumours of neural, neuroendocrine and neuroectodermal origin. They affect the oral cavity, tongue, lips, throat, nose, paranasal sinuses, larynx, salivary glands, ears and thyroid. Head and neck cancers are most common in people over 45, with the exception of nasopharyngeal carcinoma, which is characterized by the presence of two peaks of incidence between the ages of $15-35$ and above 50 . Due to the location, where all the sense organs are located in a relatively small area, a tumour in the region abundant with vascularization and innervation leads to varying degrees of dysfunction of vital signs, such as breathing, eating and speech [8].

The treatment applied in head and neck cancers are, in the first place, surgery, radiotherapy and chemotherapy. The low efficacy of the conventional methods in the treatment of tumour diseases was one of the reasons for seeking alternative therapies. The therapy using oncolytic viruses is a promising strategy for the treatment of head and neck cancers.

Adenovirus Onyx- 015 was the first modified oncolytic virus to be tested in clinical trials in cancer patients. Onyx015 was produced using genetic engineering techniques by removing the E1B $55 \mathrm{kDa}$ gene from the viral genome. The expression of the E1B-55 $\mathrm{kDa}$ adenoviral gene makes it possible for adenoviruses to replicate in the cell. The protein product of the gene binds to and inactivates cellular protein $\mathrm{p} 53$. Infections with adenoviruses with a defective E1B-55 kDa gene induce an increase in p53 and lead to cell apoptosis. P53 is a tumour suppressor protein involved in the control of many cellular processes, particularly in the activation of DNA repair mechanisms and induction of apoptosis in response to DNA damage $[9,10]$. The modified ONYX-015 virus is not capable of replication in normal cells with an active p53, whereas it does replicate in cells with non-functional p53 protein. Its intense proliferation leads to tumour cell lysis, and the released progeny viral particles infect the neighbouring tumour cells, thus leading to their destruction. Over $50 \%$ of human cancers show a nonfunctional mutant form of the p53 protein [11].

In recent years, there have been many reports in the literature for the possible use of the Onyx- 015 virus in the treatment of head and neck cancers. The presented study was conducted in patients with recurrent squamous cell carcinoma of the head and neck after surgery, radiotherapy, chemotherapy, or a combined therapy. The intratumoral administration of Onyx-015 was well tolerated and safe, with little adverse effects, mainly mild flu-like symptoms. At the same time, there was no significant tumour response. Ganly et al [12] reported the results of studies in a group of 22 patients with recurrent squamous cell head and neck carcinoma. The reduction in tumour size was observed in $14 \%$ of patients, while progression of disease was noted in $41 \%$ of patients. In another study, conducted by Nemunaitis et al $[13,14]$ on a group of 40 patients with advanced head and neck cancer, a response of the tumour was achieved in $14 \%$, while progression of disease occurred in 52\% of patients. Morley et al [15], whose study objective was not to achieve tumour regression, but to show that the administration of oncolytic virus directly to the tumour acts selectively in cancer cells without adversely affecting the healthy tissue. The study included 15 patients with oral squamous cell carcinoma, without previous treatment.

In order to increase the effectiveness of oncolytic viruses, attempts have been made to use them in combination with other methods, including the use of oncolytic viruses in combination with chemotherapy; the first clinical trials of chemotherapy combined with viral therapy were conducted in 1998. The combination of oncolytic viruses with chemotherapy can lead to synergistic effects despite different mechanisms of activity, and understanding the mechanisms of synergy will be very important for maximizing the efficacy of virus-drug therapy. Some clinical studies are now aimed at increasing the synergy between oncolytic viruses and a specific chemotherapy. Khuri et al [16] reported using intratumoral administration of Onyx- 015 with cisplatin and 5-fluorouracil in patients with recurrent squamous cell carcinoma of the head and neck. A significant response to that therapy was achieved. Over a period of 6 months, none of the tumours under study progressed.

Another adenovirus used in the treatment of head and neck cancers is E1B-55K-deleted adenovirus H101. This was the first oncolytic virus to be approved for use in combination with chemotherapy [17]. The study of patients with malignant diseases treated with intratumorally administered H101 proved to be safe. Using routine chemotherapy at the same time led to a reduction in tumour mass $[18,19,20]$. Xia et al [21] presented the results of tests carried out on a group of 160 patients who received the combination therapy with $\mathrm{H} 101$ combined with cisplatin and 5-fluorouracil, or H101 with adriamycin and 5-fluorouracil. They achieved a significant difference in response to treatment with chemotherapy versus monotherapy. Oncorine(H101), an oncolytic adenovirus similar to Onyx-015 (E1B-55K/E3-deleted), was approved in 2005 by the Chinese SFDA to be used in conjunction with chemotherapy for the treatment of nasopharyngeal carcinoma, combined with 5-FU and Cisplatin chemotherapy. This is the first oncolytic virus product approved by a governmental agency for human use [27]. 
KH901 is an oncolytic adenovirus that undergoes replication and leads to selective destruction of cells with telomerase expression; it also induces the expression of granulocyte-macrophage colony-stimulating factor (GMCSF) [22]. In contrast to normal cells, telomerase is active in most tumour cells [23]. KH901 carries the cDNA of human GM-CSF in the E3 region to express GM-CSF in cancer, which can stimulate the immune response. KH901 not only directly lyses tumour cells to generate antitumour activity in local tumours, but also stimulates a systemic antitumour immune response to distant metastases. The preliminary results on 23 patients showed that intratumoral administration of KH901 was feasible, well tolerated and associated with biological activity. Further investigation of KH901, particularly in combination with systemic chemotherapy, is necessary [22].

Another virus used in oncolytic viral therapy is HSV-1, a DNA virus and a member of the Herpesviridae family. The first genetically-engineered oncolytic virus was thymidine kinase gene-deleted virus HSV-1 [24]. Deletion of the thymidine kinase gene means that the virus can only replicate efficiently in cells with upregulation of the EGFR/Ras signalling pathway, which is commonly the case in tumour cells. Oncolytic virus HSV-1 with deletion of UL56 gene is named HF10. Intratumoral injection of HF10 into subcutaneous nodules in patients with head and neck squamous cell carcinoma caused infection and lysis of cancer cells without any significant adverse effects [25]. HSV1716 has a deletion in the RL-1 gene, which encodes the ICP34.5 protein that affects virulence. A study of 20 patients with oral cancer before surgery confirmed that such a procedure is well tolerated and without serious complications [26].

OncoVexGM-CSF is another oncolytic virus which has been developed to achieve an even more efficient anti-tumour immune responses, by the deletion of the gene encoding ICP47, a protein which inhibits antigen presentation in HSVinfected cells, and the insertion of the gene encoding the granulocyte-macrophage colony-stimulating factor (GMCSF). Onco Vex GM- CSF is well tolerated and safe [27].

Human reoviruses are naked dsRNA viruses. The type 3 Dearing oncolytic reovirus is cytotoxic to cancer cells with an overactive signalling pathway, mediated by the c-ras protein. In normal cells, the replication of reoviruses is inhibited by protein kinase, activated by dsRNA (PKR), while in the cells with overactive c-ras pathway, the activity of PKR is weakened, which makes the replication of reoviruses possible. Over $30 \%$ of human tumours have a mutation in the c-ras gene, which allows the use of specific properties of reoviruses as oncolytic agents [28].

The first reports on the possible use of Newcastle disease virus (NDV) in the treatment of cancer in humans dates back to 1964 . NDV is a paramyxovirus, avian paramyxovirus serotype 1 (PMV-1), which cause disease in animals; in humans it may cause mild flu-like symptoms. It is a singlestranded RNA virus which is capable of a much faster replication in tumour cells than in normal cells. Studies carried out using a modified NDV (F3aa)-GFP confirmed the oncolytic potential of the virus in vitro against head and neck cancer cells. Intratumour administration of NDV(F3aa)-GFP causes regression without any symptoms of toxicity [29].

Skin cancer. Skin cancer is the most common type of human cancer. During histological examination, basal cell carcinoma and squamous cell carcinoma predominate. The most common locations are the exposed parts of the body: head, neck, hands. This is related to carcinogenic UV rays. The basic treatment is surgical excision of the tumor with an adequate margin cut. In some cases, a local fluoruracil chemotherapy or radiotherapy is applied. Basal cell carcinoma is one of the locally malignant tumours, despite the ability for quick re-growth after incomplete removal, practically no metastasizes to lymph nodes and other organs occur. Whereas squamous cell carcinoma can metastasize to regional lymph nodes and metastasis via the blood stream. This occurs more frequently as the tumor is larger and more deeply invades tissue (subcutaneous tissue, adipose tissue) and concerns $2 \%-20 \%$ of cases. The most common site for metastatic squamous cell carcinoma of the skin are the lungs [30, 31].

Vahid Salimi et al [32] studied the cytotoxic effects of RSV on cell skin cancer line A431. For the tests, RSV A2 grown on human epithelial cell Hep-2 was used, and to assess the tumour cell growth inhibition the MTT test was used. In their studies they used different incubation times and different concentrations of viruses that infected cancer cells. RSV infection resulted in a significant decrease in the percentage of live A431 cells. The results indicate that RSV inhibit A431 cell viability in a dose- and time-dependent manner. The researchers could not determine the exact mechanisms of destruction of cancer cells by RSV. In other tumour types, the anticancer properties of this virus are also not clearly defined. It is known that RSV can induce apoptosis in cells of the respiratory epithelium via specific stress-activated caspase (caspase-12). It has been shown that in RSV-induced disorders, selective PC-3 human prostate cancer cells are created by the down-regulation of NF-kB and stimulate intrinsic pathway of apoptosis [33]. However, data on the impact of RSV on apoptosis in other cell types are incompatible. Some studies suggest that apoptosis of neutrophils can be accelerated in the bronchioles of the ongoing RSV infection. This is explained by the influence of modulating RSV on neutrophils which are in contact with the virus in the damaged airway epithelial cells. Other studies have shown that RSV can cause delay apoptosis of neutrophils and eosinophils by phosphatidylinositol 3-kinase (PI3K) and NF-kB related pathways, including up-regulation of anti-apoptotic factors. Furthermore, it has been shown that RSV inhibits neutrophil apoptosis by activation of PI3-K, NF$\mathrm{kB}$ and $\mathrm{p} 38$, by intercellular interaction with TLR. [34]. The encouraging results of in vitro tests require further studies; however, the use of RSV in the treatment of cancers of the skin gives a great opportunity and promise as an alternative method of treatment.

Hepatocellular carcinoma. Hepatocellular carcinoma is a tumour derived from hepatocytes, and most often formed in cirrhotic liver as a result of HBV, HCV or HDV infection. Less frequent risk factors are haemochromatosis, aflatoxin poisoning, and alcoholic liver disease, among others. The primary method of treatment is a liver transplant in patients eligible for surgery. Sorafenib has been recently introduced for the treatment (kinase inhibitor with antiangiogenic and antiproliferative mechanisms of action). In the absence of treatment the mean survival is approximately 3 months. In the case of removing the tumour without transplant, there is a 5 year survival rate of $25 \%$; after liver transplant, about 
$80 \%$. Patients after liver transplantation require a lifetime of immunosuppressive drugs to reduce the risk of liver rejection. This creates a high risk of susceptibility to infectious diseases and requires large financial resources. Therefore, it is important to effectively search for new diagnostic methods [35]. Attempts have been made to use oncolytic viruses for the treatment of hepatocellular carcinoma.

An example of the use of new therapy are studies involving HSV-1 G207. This is an attenuated, unable to replicate, second-generation virus strain derived from R3616. The replicative activity is suppressed by the deletion of one copy of the gene diploid ICP 6 and deletion of both copies of the $\gamma 1$ 34.5 neurovirulence gene [36]. The virus has the introduced gene of E. coli $\beta$-galactosidase (LacZ), the UL39 gene which serves as a marker for infection. Disruption of the gene UL39 eliminates nucleotide reductase (RR), increasing the activity and specificity of the virus into cells, such as tumour cell proliferation. Tae-Jin Song and al [37] studied the effects of the virus on the G207 in inhibiting the development of liver cancer cell lines. In their research they used the following cell lines: Hep 3B2.1-7 (Hep3B, Hep 3B, Hep-3B, HB-8064), Hep G2 (HepG2, HB 8065), PLC/PRF/5 (PLC5 CRL-8024) i SK-HEP-1 (HTB-52), SNU-182, SNU-354, SNU-368, SNU387, SNU-398, SNU-423, SNU-449, SNU-475, SNU-739, SNU-761, SNU -878 i SNU-886. On the most of cell lines, apparent cytotoxicity of the virus was observed: HepG2, PLC5 i Hep3B after 7-day incubation, almost 100\% growth inhibition was achieved, while in the SNU-182, SNU-387, SNU-886, SNU-878 the cytotoxicity of the virus was more than 70\%. Lines SK-HEP-1, SNU-398, SNU-475, SNU-354 and SNU-423 were completely resistant to the virus.

Vaccinia virus JX-594 is another example of using viruses to fight against hepatocellular carcinoma. Genetic material was modified by adding the gene responsible for the formation of GM-CSF causing potentiation of antitumour activity of the host. Otherwise removed genes responsible for the formation of thymidine kinase. This modification prevents replication in non-malignant cells, but it may occur in cells which are activated EGFR. This increases the specificity of therapy. Virus replication results in cells lysis [38]. The researchers also studied the interaction of the use of JX-594 in combination with sorafenib. Although the study was conducted on a very small group of patients, in the absence of major side-effects, the results appear to be very promising [39].

Bladder Cancer. This is a cancer that occurs more often in people of advanced age, and more than three times more common in men than women. Histologically it usually comes from the transitional epithelium of the bladder covering; at present they are called urothelial neoplasms, and are a very rare squamous cell carcinoma [40]. Cigarette smoking is a very important carcinogenic factor, other factors include exposure to chemical compounds, mainly from the group of aromatic amines, and chronic inflammation of the bladder. The basic treatment is surgical removal of the tumour, often together with the entire bladder. Palliative chemotherapy is the treatment that prolongs life, reduces pain, but does not lead to recovery. The treatment is used as an immunotherapy which involves the administration of BCG vaccine intravesically. Tuberculous mycobacteria strongly induce an immune response associated with apoptosis [41]. The prognosis for bladder cancer depends on the stage, and with removal of the bladder, a 5 -year survival rate is about 20\% [42]. Virotherapy in the case of this cancer has good prospects ahead. The location creates good conditions for administering viruses to the tumour, thereby avoiding potentially adverse effects that may result from systemic administration and enables the supply of the drug under pattern control. The characteristic structure of these tumours (mostly papillary shape) allows increasing the space available after the intravesical injection, and the efficacy of the BCG treatment increases the chances of efficacy of the viruses. For this reason, many attempts are being undertaken using different viruses, with quite positive results.

Hanel et al [43] evaluated the efficacy of retroviral therapy in mouse models of bladder tumour. Analysis of the surviving animals treated with viral therapy and compared to the control group in which used of BCG therapy, showed that after 100 days, $90 \%$ of the animals treated with retroviruses were alive, compared to only $50 \%$ of control group.

Glomel et al [44] focused on the use of vaccinia virus vaccination in patients scheduled for removal of the bladder. Patients received three doses of viruses 24 hours after the patients underwent surgery. The microscope slides observed significant swelling of the mucous membrane and widening of blood vessels. Cancer and normal urothelial cells showed evidence of viral infection, i.e. enlarged cells with the swollen cytoplasmic vacuoles. This may indicate that the viruses could start the inflammatory process leading to the destruction of tumour cells.

Ramesh et al [45] studied the use of CG0070 virus in the treatment of bladder cancer. CG0070 is a strain of adenovirus wherein for the expression of the viral gene E1A is required human transcription factor E2F-1. It is present in bladder cancer cells. Furthermore, CG0070 encodes human stimulating factor GM-CSF, which stimulates the maturation of cytokines, recruitment of macrophages and dendritic cells. All these elements are a potent inducer of the local anti-tumour immunity. Selectivity CG0070 was associated with a 100-fold greater replication and 1,000-fold greater cytotoxicity in the bladder TCC cells, compared to normal human fibroblasts. CG0070 was tested for bladder cancer cells in mouse models with a human tumour xenografts bladder. A significant antitumour effect was observed after five injections of CG0070 in concentrations up to $3 \times 10^{\wedge}$ 10 virus particles per dose. In a half of the mice ( 5 out of 10 ) which received the highest dose, analysis showed complete regression of the tumour compared to no regression in mice in the control group treated with standard therapy (PBS). These promising preclinical data led to a phase I clinical trial of CG0070, focusing on the treatment of patients with recurrent bladder cancer after treatment with BCG. The results of single and multiple dose treatment (weekly or monthly 3x 6x) using CG0070 given intravesically at doses up to $10 \wedge 13$ particles of virus in 35 patients demonstrated a response rate of $23 \%$ in a single dose and $64 \%$ in the multidose groups. Quite a large efficacy associated with minor side-effects in the form of a small haematuria and symptoms of dysuria [46].

Prostate Cancer. Prostate cancer is a frequently occurring cancer among men. The incidence of the disease increases with age, the peak prevalence in the 65-75 years of age group. An adenocarcinomas account for almost $80 \%$ of all cases a prostate tumour. The most important factors leading to malignant transformation include hormonal factors 
(androgens), genetic predisposition, race (especially the black race), as well as environmental factors, such as a diet high in animal fat. The cancer metastasizes quickly, especially to the bone. The primary method of treatment is surgery. As a complement, radiotherapy is applied. Due to the important role of male hormones for tumour formation, hormones with antagonist activity to them are used in the therapy, and as palliative treatment, chemotherapy is given. Although there are many therapies, the 5-year survival rate is about 65\% [47].

In the presented study, researching for an alternative procedure, adenoviruses were used. ONYX-15 strain is deprived of the E1B-55K gene. With this modification, the replication can take place only in cells with a mutation in the gene responsible for the synthesis of the p53 protein. Prostate cancer cells meet this condition and sets a good example for this strain of the virus. Another subject of the study was the role of the Ad5 type CV706. It has a change in the genetic material $\mathrm{E} 1$ and needs to replicate a prostatespecific promoter (PSP). Thus, expression of E1 proteins will be strictly confined to the prostate tissue [48]. Newcastle disease virus (NDV) application of this virus is particularly advantageous because under normal conditions it does not cause infection in humans. Phuangsab et al [49] in research on NDV and prostate cancer cells recorded a reduction of a growth at the level $77-96 \%$.

\section{CONCLUSION}

The constant increase in the incidence of cancer requires continuous improvement in oncological treatment. Traditional methods carry the risk of adverse effects on all systems and organs in the body, and their efficiency is often too low. In many cases, after determining the severity of the neoplastic process, the only procedure that can be offered to a person suffering from cancer is a symptomatic palliative care which reduces pain and slightly increasing the quality of life. It does not give any chance for healing. Therefore, it is necessary to search for new and alternative methods of cancer treatment. Oncolytic viruses carry high hopes of improving survival of oncological treatment. Although the anticancer effects of a virus has been observed since the beginning of the twentieth century, the therapy of oncolytic virus is currently undergoing a renaissance. Evidenced by the large amount of research, many publications present good prospects for the use of many types of viruses in the treatment. An important positive argument is being allowed to take the treatment to a significant degree of severity of the cancer, and distant metastases, often disqualifying traditional methods of treatment. Another advantage of this therapy is the ability to use it synergistically with chemotherapy, radiotherapy, or as a preparation before surgery (reduction of tumou size). However, it must not be forgotten that many constraints need to be overcome before considering this method as being effective and accessible to patients. Currently, most of the studies carried out in vitro are on animals. Using cell lines eliminates the immune system which in living organisms can neutralize the viruses before penetration into the tumour cells. Direct administration intratumorally is not always possible. An important problem is to increase the virus's specificity for cancer cells, so as not to cause lysis of normal cells. This is a particularly important issue for tumours located near important organs, such as the brain or blood flow, as well as theliver. Potential risks may also concern mutations that occur during replication of viruses, and quite often occur due to the low efficiency of virus repair systems. This could lead to the creation of new strains that can infect in an uncontrolled way healthy, correctly functioning cells and lead to a generalized infection. Exact understanding the mechanisms of anti-tumour activity oncolytic viruses requires detailed immunological, genetic, and biochemical studies. This generates significant costs for oncolytic virus therapy, but they are necessary to improve efficiency and eliminate the toxicity of the therapy. Despite these concerns, the use of viruses for cancer therapy gives great hope of further research in this direction and the effectiveness of providing an ever-increasing group of oncolytic viruses used for new types and strains, as well as steady progress in clinical trials. The efficacy of oncolytic virus therapy is justified by the continuous increase in the group of oncolytic virus, new types and strains, and the continued progress in clinical trials.

\section{REFERENCES}

1. Alemany R. Viruses in cancer treatment. Clin Transl Oncol. 2013; 15(3): 182-188.

2. Mullen T, Tanabe K. Viral oncolysis. The Oncol. 2002; 7: 1-6: 119

3. Wojton J, Kaur B. Impact of tumor microenvironment on oncolytic viral therapy. Cytokine Growth Factor Rev. 2010; 21(2-3): 127-134.

4. Singh PK, Doley J, Kumar GR, Sahoo AP, Tiwari AK. Oncolytic viruses \& their specific targeting to tumour cells. Indian J Med Res. 2012;136(4): 571-584.

5. Naik JD, Twelves CJ, Selby PJ, Vile RG, Chester JD.Immune recruitment and therapeutic synergy: keys to optimizing oncolytic viral therapy? Clin Cancer Res. 2011; 17(13): 4214-4224.

6.Zeyaullah M, Patro M, Ahmad I, Ibraheem K, Sultan P, Nehal M, Ali A. Oncolytic viruses in the treatment of cancer: a review of current strategies. Pathol Oncol Res. 2012; 18(4): 771-781.

7. Tong AW, Senzer N, Cerullo V, Templeton NS, Hemminki A, Nemunaitis J. Oncolytic viruses for induction of anti-tumor immunity. Curr Pharm Biotechnol. 2012; 13(9): 1750-1760.

8. Rusin P, Markiewicz Ł, Majsterek I. Uwarunkowania genetyczne nowotworów głowy i szyi. Post Hig Med Dosw. 2008; 62: 490-501.

9. Lane DP. p53, guardian of the genome. Nature (Lond). 1992; 358: 15-16.

10. Levine AJ. p53 the cellular gatekeeper for growth and division. Cell. 1997; 88: 323-331.

11. Hollstein M, Sidransky D, Vogelstein B, Harris C. p53 mutations in human cancer. Science (Washington DC). 1991; 49: 49-53.

12. Ganly I, Kirn D, Eckhardt G, Rodriguez GI, Soutar DS, Otto R i wsp. A Phase I study of Onyx-015, an E1B attenuated adenovirus, administered intratumorally to patients with recurrent head and neck cancer. Clin Cancer Res. 2000; 6: 798-806.

13. Nemunaitis J, Ganly I, Khuri F, Arseneau J, Kuhn J, McCarty T i wsp. Selective replication and oncolysis in p53 mutant tumors with ONYX015, an E1B-55 kDa gene-deleted adenovirus, in patients with advanced head and neck cancer: a Phase II trial. Cancer Res. 2000; 60: 6359-6366.

14. Nemunaitis J, Khuri F, Ganly I, Arseneau J, Posner M, Vokes E i wsp. Phase II trial of intratumoral administration of ONYX-015, a replication-selective adenovirus, in patients with refractory head and neck cancer. J Clin Oncol. 2001; 19: 289-298.

15. Morley S, MacDonald G, Kirn D, Kaye S, Brown R, Soutar D i wsp. The dl1520 virus is found preferentially in tumor tissue after direct intratumoral injection in oral carcinoma. Clin Cancer Res. 2004; 10: 4357-4362.

16. Khuri FR, Nemunaitis J, Ganly I, Arseneau J, Tannock IF, Romel Li wsp. A controlled trial of intratumoral ONYX-015, a selectively-replicating adenovirus, in combination with cisplatin and 5-fluorouracil in patients with recurrent head and neck cancer. Nat Med. 2000; 6: 879-885.

17. Wong HH, Lemoine NR, Wang Y. Oncolytic Viruses for Cancer Therapy:Overcoming the Obstacles.Viruses. 2010; 2(1): 78-106.

18. Yuan ZY, Zhang L, Li S, Qian XZ, Guan ZZ. Safety of an E1B deleted adenovirus administered intratumorally to patients with cancer. Ai Zheng. 2003; 22: 310-313. 
19.Xu RH, Yuan ZY, Guan ZZ, Cao Y, Wang HQ, Hu XH i wsp. Phase II clinical study of intratumoral H101, an E1B deleted adenovirus, in combination with chemotherapy in patients with cancer. Ai Zheng. 2003; 22: 1307-1310.

20. Lu W, Zheng S, Li XF, Huang JJ, Zheng X, Li Z. Intra-tumor injection of $\mathrm{H} 101$, a recombinant adenovirus, in combination with chemotherapy in patients with advanced cancers: a pilot Phase II clinical trial. W J Gastroenterol. 2004; 10: 3634-3638.

21. Xia ZJ, Chang JH, Zhang L, Jiang WQ, Guan ZZ, Liu JW i wsp. Phase III randomized clinical trial of intratumoralinjection of E1B gene-deleted adenovirus (H101) combined with cisplatin-based chemotherapy in treating squamous cell cancer of head and neck or esophagus. Ai Zheng. 2004; 23: 1666-1670.

22. Chang J, Zhao X, Wu X, Guo Y, Guo H, Cao J i wsp. A Phase I study of KH901, a conditionally replicating granulocyte-macrophage colonystimulating factor: armed oncolytic adenovirus for the treatment of head and neck cancers. Cancer Biol Ther. 2009; 8(8): 676-682.

23. Kowalska A, Kowalik A. Telomer i telomeraza w onkogenezie. Współcz. Onkol. 2006; 10(10): 485-496.

24. Wodarz D, Hofacre A, Lau JW, Sun Z, Fan H, Komarova NL. Complex spatial dynamics of oncolytic viruses in vitro: mathematical and experimental approaches. PloS Comput Biol. 2012; 8(6): e1002547.

25. Fujimoto Y, Mizuno T, Sugiura S, Goshima F, Kohno S, Nakashima T i wsp. Intratumoral injection of herpes simplex virus HF10 in recurrent head and neck squamous cell carcinoma. Acta Otolaryngol. 2006; 126: 1115-1117.

26. Mace AT, Harrow SJ, Ganly I, Brown SM. Cytotoxic effects of the oncolytic herpes simplex virus HSV 1716 alone and in combination with cisplatin in head and neck squamous cell carcinoma. Acta Otolaryngol. 2007; 127: 880-887.

27. Hu JC, Coffin RS, Davis CJ, Graham NJ, Groves N, Guest PJ i wsp. A phase I study of OncoVEXGM-CSF, a second-generation oncolytic herpes simplex virus expressing granulocyte macrophage colonystimulating factor. Clinical Cancer Research. 2006; 12(22): 6737-6747.

28. Kyula JN, Roulstone V, Karapanagiotou EM, Melcher AA, Harrington KJ. Oncolytic reovirus type 3 (Dearing) as a novel therapy in head and neck cancer. Expert Opin Biol Ther. 2012; 12: 1669-1678.

29. Li P, Chen CH, Li S, Givi B, Yu Z, Zamarin D i wsp. Therapeutic effects of a fusogenic newcastle disease virus in treating head and neck cancer. Head Neck. 2011; 33(10): 1394-1399.

30. moje! DeAmbrosis K, De'Ambrosis B. Nonmelanoma skin cancer with perineural invasion: report of outcomes of a case series. Dermatol Surg. 2010;36(1):133-138.

31. Mueller CS, Reichrath J. Histology of melanoma and nonmelanoma skin cancer. Adv Exp Med Biol. 2008; 624: 215-226.

32. Salimi V, Tavakoli-Yaraki M, Mahmoodi M, Shahabi S, Gharagozlou M, Shokri F, Mokhtari-Azad T. The Oncolytic Effect of Respiratory Syncytial Virus (RSV) in Human Skin Cancer Cell Line, A431. Iran Red Crescent Med J. 2013 January; 15(1): 62-67.

33. Echchgadda I, Kota S, DeLa Cruz I, Sabbah A, Chang T, Harnack R, et al. Anticancer oncolytic activity of respiratory syncytial virus. Cancer Gene Ther. 2009; 16(12): 923-35
34. Echchgadda I, Kota S, DeLa Cruz I, Sabbah A, Chang T, Harnack R, et al. Anticancer oncolytic activity of respiratory syncytial virus. Cancer Gene Ther. 2009; 16(12): 923-935.

35. El-Serag HB. Epidemiology of viral hepatitis and hepatocellular carcinoma. Gastroenterology. 2012; 142(6): 1264-1273.

36. Whitley RJ, Kern ER, Chatterjee S, Chou J, Roizman B. Replication, establishment of latency, and induced reactivation of herpes simplex virus gamma 134.5 deletion mutants in rodent models. Journal Of Clinical Investigation. 1993; 91: 2837-2843

37. Tae-Jin Song D Eisenberg P Adusumilli M Hezel Y Fong. Oncolytic Herpes Viral Therapy is Effective in the Treatment of Hepatocellular Carcinoma Cell Line J. Gastrointest Surg. 2006; 10(4): 532-542.

38. K Parato C J Breitbach F Le Boeuf J Wang Ch Storbeck C Ilkow et al. The Oncolytic Poxvirus JX-594 Selectively Replicates in and Destroys Cancer Cells Driven by Genetic Pathways Commonly Activated in Cancers Mol Ther. 2012; 20(4): 749-758.

39. J Heo C J Breitbach A M Chang W Kim R Patt M K Kim Sequential Therapy With JX-594, A Targeted Oncolytic Poxvirus, Followed by Sorafenib in Hepatocellular Carcinoma: Preclinical and Clinical Demonstration of Combination Efficacy Mol Ther. 2011; 19(6): 11701179.

40. Kachuri L, De P, Ellison LF, Semenciw R. Cancer incidence, mortality and survival trends in Canada,1970-2007. Chronic Dis Inj Can. 2013; 33(2): 69-80.

41. Paliwal P, Arora D, Mishra AK. Epithelial mesenchymal transition in urothelial carcinoma: twist in the tale. Indian J Pathol Microbiol. 2012; 55(4): 443-449.

42. Matteo Fassan, Edouard J Trabulsi, Leonard G Gomella, Raffaele Baffa Targeted therapies in the management of metastatic bladder cancer. Biologics. 2007; 1(4): 393-406

43. Hanel EG, Xiao Z, Wong KK, LeePW, Britten RA, Moore RB. A novel intravesical therapy for superficial bladder cancer in an orthotopic model: oncolytic reovirus therapy. J Urol. 2004 Nov;172(5 Pt 1): 2018 2022.

44. Gomella LG, Mastrangelo MJ, McCue PA, Maguire HC JR, Mulholland SG, Lattime EC. Phase i study of intravesical vaccinia virus as a vector for gene therapy of bladder cancer. J Urol. 2001; 166(4): 1291-1295.

45. Ramesh N, Ge Y, Ennist DL, Zhu M, Mina M, Ganesh S, Reddy PS, Yu DC. CG0070, a conditionally replicating granulocyte macrophage colony-stimulating factor--armed oncolytic adenovirus for the treatment of bladder cancer. Clin Cancer Res. 2006; 12(1): 305-313.

46. Senzer N, Nemunaitis J, Goldstein M, et al. A phase 1 dose-escalation trial of intravesical CG0070 for superficial Transitional Cell Carcinoma (TCC) of the Bladder after Bacillus Calmette-Guerin (BCG) failure. Molecular Therapy. 2006; 13.

47. Tsivian M, Abern MR, Polascik TJ. Evolution of the concept of focal therapy for prostate cancer. Oncology (Williston Park). 2013; 27(1): $64-68,70$.

48. Mullen JT, Tanabe KK.Viral oncolysis. Oncologist. 2002; 7(2): 106-119.

49. Phuangsab A, Lorence RM, Reichard KW, Peeples ME, Walter RJ. Newcastle disease virus therapy of human tumor xenografts: antitumor effects of local or systemic administration. Cancer Lett. $2001 ; 172: 27-36$. 